\title{
Cell Type-Specific Oxidative Stress Genomic Signatures in the Globus Pallidus of Dopamine-Depleted Mice
}

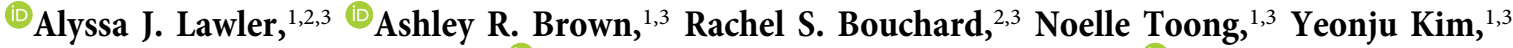 \\ Nitinram Velraj, ${ }^{1,3}$ Grant Fox, ${ }^{1,3}{ }^{-}$Michael Kleyman, ${ }^{1,3}$ Byungsoo Kang, ${ }^{1,3}{ }^{-1}$ Aryn H. Gittis, ${ }^{2,3}$ and \\ (1)Andreas R. Pfenning ${ }^{1,3}$ \\ ${ }^{1}$ Computational Biology, ${ }^{2}$ Biological Sciences, and ${ }^{3}$ Neuroscience Institute, Carnegie Mellon University, Pittsburgh, Pennsylvania 15213
}

Neuron subtype dysfunction is a key contributor to neurologic disease circuits, but identifying associated gene regulatory pathways is complicated by the molecular complexity of the brain. For example, parvalbumin-expressing $\left(\mathrm{PV}^{+}\right)$neurons in the external globus pallidus (GPe) are critically involved in the motor deficits of dopamine-depleted mouse models of Parkinson's disease, where cell type-specific optogenetic stimulation of $\mathrm{PV}^{+}$neurons over other neuron populations rescues locomotion. Despite the distinct roles these cell types play in the neural circuit, the molecular correlates remain unknown because of the difficulty of isolating rare neuron subtypes. To address this issue, we developed a new viral affinity purification strategy, Cre-Specific Nuclear Anchored Independent Labeling, to isolate Cre recombinase-expressing $\left(\mathrm{Cre}^{+}\right)$nuclei from the adult mouse brain. Applying this technology, we performed targeted assessments of the cell type-specific transcriptomic and epigenetic effects of dopamine depletion on $\mathrm{PV}^{+}$and $\mathrm{PV}^{-}$cells within three brain

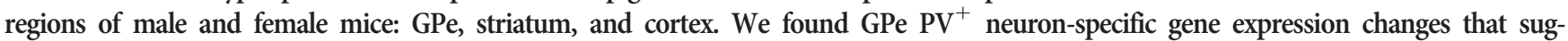
gested increased hypoxia-inducible factor $2 \alpha$ signaling. Consistent with transcriptomic data, regions of open chromatin affected by do-

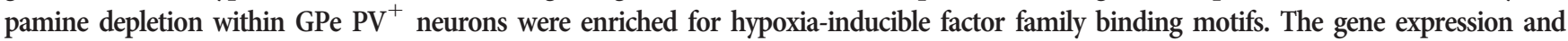
epigenomic experiments performed on $\mathrm{PV}^{+}$neurons isolated by Cre-Specific Nuclear Anchored Independent Labeling identified a transcriptional regulatory network mediated by the neuroprotective factor Hif2a as underlying neural circuit differences in response to dopamine depletion.

Key words: epigenomics; globus pallidus; Hif2a; oxidative stress; Parkinson’s disease; parvalbumin

Significance Statement

Cre-Specific Nuclear Anchored Independent Labeling is an enhanced, virus-based approach to isolate nuclei of a specific cell type for transcriptome and epigenome interrogation that decreases dependency on transgenic animals. Applying this technology to GPe parvalbumin-expressing neurons in a mouse model of Parkinson's disease, we discovered evidence for an upregulation of the oxygen homeostasis maintaining pathway involving Hypoxia-inducible factor $2 \alpha$. These results provide new insight into how neuron subtypes outside the substantia nigra pars compacta may be compensating at a molecular level for differences in the motor production neural circuit during the progression of Parkinson's disease. Furthermore, they emphasize the utility of cell type-specific technologies, such as Cre-Specific Nuclear Anchored Independent Labeling, for isolated assessment of specific neuron subtypes in complex systems.

Received June 25, 2020; revised Aug. 23, 2020; accepted Aug. 27, 2020.

Author contributions: A.J.L., A.R.B., A.H.G., and A.R.P. designed research; A.J.L., A.R.B., R.S.B., N.T., Y.K., N.V., and G.F. performed research; A.J.L. contributed unpublished reagents/analytic tools; A.J.L., N.T., N.V., M.K., and B.K. analyzed data; A.J.L. wrote the first draft of the paper; A.J.L., A.R.B., A.H.G., and A.R.P. edited the paper; A.J.L. and A.R.P. wrote the paper.

This material is based upon work supported by NIH Grant DP1DA046585 and the National Science Foundation Graduate Research Fellowship Grants DGE1252522 and DGE1745016. We thank Irene Kaplow for implementing the ATAC-seq analysis pipeline; Jenna Schwenk, Naomi Shin, Morgan Wirthlin, BaDoi Phan, Alexander Chamessian, and David Gallegos for experimental support; and Alison Barth, Anne E. West, and Victor T. Molina for helpful discussions.

The authors declare no competing financial interests.

Correspondence should be addressed to Alyssa J. Lawler at alawler@andrew.cmu.edu or Andreas R. Pfenning at apfenning@cmu.edu.

https://doi.org/10.1523/JNEUROSCI.1634-20.2020

Copyright $\odot 2020$ Lawler et al.

This is an open-access article distributed under the terms of the Creative Commons Attribution 4.0 International license, which permits unrestricted use, distribution and reproduction in any medium provided that the original work is properly attributed.

\section{Introduction}

A unique challenge for identifying pathologic gene regulation in neurologic diseases is the extreme heterogeneity of brain tissues, which are often comprised of dozens of molecularly distinct cell types and neuron subtypes (Lake et al., 2018; Saunders et al., 2018; Tasic, 2018). Even abnormal expression of a single gene within a minority cell population can exert outsized effects on the system (Wen et al., 2010; Dutton et al., 2013). One neuron subtype important in many diseases is fast-spiking parvalbuminexpressing $\left(\mathrm{PV}^{+}\right)$neurons, which are essential for maintaining balance between excitatory and inhibitory influences brainwide (Hu et al., 2014; Ferguson and Gao, 2018).

In the basal ganglia, $\mathrm{PV}^{+}$neurons are optimally positioned to regulate the motor circuits involved in movement disorders, 
such as Parkinson's disease (PD). $\mathrm{PV}^{+}$neurons constitute roughly half of all neurons in the GPe, a key contributor to pathogenic hypersynchrony of basal ganglia nuclei under the dopaminedepleted conditions of PD (Bergman et al., 1998; Bevan et al., 2002; Mallet et al., 2008). GPe $\mathrm{PV}^{+}$neurons, also called prototypical neurons, have fast, regular firing properties and strongly project to the subthalamic nucleus, the major output nucleus for action (Hegeman et al., 2016; Saunders et al., 2016). Behaviorally, optogenetic stimulation of GPe $\mathrm{PV}^{+}$neurons, but not global GPe stimulation, has prokinetic effects in mice with late-stage dopamine depletion (DD) (Mastro et al., 2017). Additionally, reduced numbers of GPe $\mathrm{PV}^{+}$neurons have been reported in postmortem brain tissue of PD patients and certain animal models of PD (Hardman and Halliday, 1999; Fernández-Suárez et al., 2012).

Despite the associations between $\mathrm{PV}^{+}$neurons and $\mathrm{PD}$, the molecular correlates of disease in these and other neuron populations are understudied because of technical barriers. Bulk tissue RNA sequencing (RNA-seq) (Wang et al., 2009) and the assay for transposase-accessible chromatin (ATAC-seq) (Buenrostro et al., 2013, 2015) are ideal for identifying gene expression and chromatin landscapes in homogeneous tissues, and together provide a rich snapshot of gene regulation. With increased heterogeneity, though, the interpretability of bulk tissue assays breaks down because subpopulations exist in different transcriptomic and epigenetic states and experience independent changes.

In recent years, the need for molecular access to specific cell populations has triggered rapid technology development, but there are particular shortcomings pertinent to neuroepigenetics that have yet to be optimized. Manual dissection, laser capture microdissection, and FACS-based isolation technologies have all been applied toward transcriptomic profiling of isolated populations, including certain groups of $\mathrm{PV}^{+}$neurons (Sugino et al., 2006; Pietersen et al., 2014; Enwright et al., 2018). Pairing these sorting techniques with ATAC-seq has been suitable for broad, abundant cell classes in the brain (Fullard et al., 2017; Nott et al., 2019), but remains challenging for small populations because of nuclei fragility and low yield (Handley et al., 2015; Mo et al., 2015). Given the rarity of neurons compared with glia in the GPe (von Bartheld et al., 2016), these sorting methods are unlikely to produce high-quality epigenomic data.

A more recent nuclei isolation approach with advantages in epigenomics compatibility is INTACT. This method, which induces cell type-specific expression of a nuclear surface tag, was first developed in plants (Deal and Henikoff, 2010) and later adapted for murine neuronal subtypes, including $\mathrm{PV}^{+}$neurons (Mo et al., 2015). INTACT labeling can be used in combination with FACS (Chamessian et al., 2018) or with immunopurification, which is more gentle and effective for rare cell types (Handley et al., 2015; Mo et al., 2015). Relative to emerging single nucleus RNA-seq and ATAC-seq technologies, targeted profiling of specific labeled cell populations with INTACT is more efficient, especially if the population of interest is rare. Still, INTACT requires double-transgenic mice, which are time-consuming to maintain and may not be compatible with conditional disease models. To relieve some of this burden, we developed a viral INTACT system for isolating nuclei: Cre-Specific Nuclear Anchored Independent Labeling (cSNAIL). This technology combines the ubiquity of cell type-specific Cre transgenic animals with the convenience of adeno-associated viruses (AAVs). cSNAIL is particularly well suited for rare neuron subtype isolation, and the principles can scale beyond the means of transgenic technologies.

Here, we used cSNAIL to interrogate whether DD induces gene expression and chromatin accessibility changes in $\mathrm{GPe} \mathrm{PV}^{+}$ neurons that might reflect or contribute to the pathology of PD. In parallel, we conducted RNA-seq and ATAC-seq on cSNAILisolated $\mathrm{PV}^{+}$populations and the remaining $\mathrm{PV}^{-}$cells from 6-OHDA-lesioned mice and sham controls. In order to determine the extent to which DD-induced gene regulatory effects in

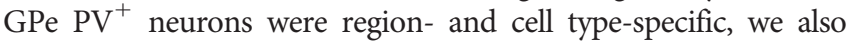
assessed $\mathrm{PV}^{+}$neurons and $\mathrm{PV}^{-}$cells in two additional regions of differing proximities to GPe, the striatum and cortex. With this strategy, we identified $\mathrm{GPe} \mathrm{PV}^{+}$neuron-specific epigenetic and transcriptional responses to DD that were related to cellular oxygen sensing and oxidative stress, providing new insight into the molecular consequences of DD.

\section{Materials and Methods}

Experimental design. The main experiments were designed to assess gene expression and chromatin accessibility signatures in cSNAIL-isolated cell populations from mice with normal and impaired dopamine production. A cohort of male littermates $(N=3)$ and a cohort of female littermates $(N=3)$, all adult heterozygous Pvalb-2A-Cre mice, were transduced with cSNAIL AAV to label $\mathrm{PV}^{+}$neurons. After 2-3 weeks of incubation, the animals underwent surgery for either a 6-OHDA injection to induce DD ( 2 males, 2 females) or a comparable saline injection ( 1 male, 1 female). This control allowed us to minimize possible confounding effects from the surgical procedure and isolate the effects of dopamine depletion itself. Three to five days after surgery, tissue from the cortex, striatum, and GPe was collected and sorted into labeled $\left(\mathrm{PV}^{+}\right)$ nuclei and unlabeled nuclei via affinity purification with cSNAIL. The six nuclei populations from each animal were then divided for ATACseq or RNA-seq. For more metadata information, see Extended Data Figure 2-1. Supporting experiments included image analysis of cSNAIL expression and antibody staining of a candidate protein involved in the response to $\mathrm{DD}$.

$A A V$ design. Several modifications were made to the INTACT system to allow it to be delivered using AAV. (1) We used an N-truncated version of the Mus musculus Sun1 gene (Sad1 and UNC84 domain-containing 1, transcript variant 5, NCBI Reference Sequence: NM_001256118.1), amino acids 208-757, that retains the ability to localize to the nuclear envelope in a manner that is resistant to detergent tissue homogenization and is similar to the variant described by Haque et al. (2010). (2) We incorporated only the DNA sequence of exonic regions of Sun1. (3) The Sun1GFP fusion gene included one copy of superfolder GFP instead of two. (4) We adjusted the Cre-dependent mechanism from a loxp-flanked stop codon to the double-inverted orientation (DIO) system. In the initial cSNAIL genome, the Sun1GFP gene is in the reverse orientation with respect to the promoter such that the gene cannot be expressed. When the virus encounters Cre recombinase protein (Cre), it acts on the double-flanking lox sites to excise the Sun1GFP gene and reinsert it in the correct orientation for transcription (see Fig. 1A).

Vector construction. The modified Sun1GFP fusion sequence fragment was synthesized by Integrated DNA Technologies and contained flanking restriction sites for SgsI and BspoI. The Sun1GFP sequence was inserted into an AAV DIO backbone vector with an Efla promoter using standard restriction cloning. pAAV-Efla-DIO-EGFP-WPRE-pA was a gift from Bernardo Sabatini (http://addgene.org/; 37084; RRID: Addgene_37084) (Saunders et al., 2012). The ligation was transformed into electrocompetent bacteria from which we selected positive clones and confirmed the sequence with Sanger sequencing. The cSNAIL genome vector pAAV-Efla-DIO-Sun1GFP-WPRE-pA is available on Addgene; RRID:Addgene_160141.

$A A V$ production. The AAV was produced by triple cotransfection of pAAV-Ef1a-DIO-Sun1GFP-WPRE-pA, an AAV helper plasmid, and pUCmini-iCAP-PHP.eB into AAVpro(R) 293T cells (Takara, catalog \#632273). pUCmini-iCAP-PHP.eB was a gift from Viviana Gradinaru (http://addgene.org/; 103005; RRID:Addgene_103005) (Chan et al., 2017). After cell expansion, the AAV particles were precipitated with polyethylene glycol and purified with ultracentrifugation on an iodixanol gradient. The virus was titrated using the $A A V \operatorname{pro}(\mathrm{R})$ Titration Kit 
(Takara, catalog \#6233), diluted in PBS to a concentration of $8.0 \times 10^{9} \mathrm{vg} / \mu \mathrm{l}$, and stored at $-80^{\circ} \mathrm{C}$ until injection.

Animals. All molecular experiments were performed on 9- to 12-week-old heterozygous Pvalb$2 A$-Cre mice (B6.Cg-Pvalb ${ }^{\text {tm1.1(cre)Aibs } / J ; ~ J a c k s o n ~}$ stock \#012358) (Madisen et al., 2010) on a C57BL/ $6 \mathrm{~J}$ background. Imaging experiments for the validation of cSNAIL (see Fig. 1) were performed with double-transgenic Pvalb-2A-Cre and Ai14 mice (Ail4 strain; B6.Cg-Gt(ROSA)26Sor ${ }^{\text {tm14(CAG-tdTomato)Hze } / \text {; }}$ Jackson stock \#007914) (Madisen et al., 2010). Experiments related to Hif2a staining (see Fig. 4D) were conducted on WT mice (C57BL/6); Jackson stock \#000664).

$A A V$ delivery. Mice were anesthetized with isoflurane for 2-5 min until breathing slowed and the animal had no pedal reflex. We injected $4 \times 10^{11} \mathrm{vg}$ of AAV into the retro-orbital cavity. The mice received $0.5 \%$ proparacaine hydrochloride ophthalmic solution for comfort and were monitored for physical and behavioral abnormalities after procedure, but none were observed. The virus incubated for 3-5 weeks to reach peak expression before downstream experiments.

Dopamine depletion surgery. The molecular experiments were designed to reproduce previous conditions and maximize cell yield. Therefore, we induced bilateral acute 6-OHDA or saline injections to the medial forebrain bundle as described by Mastro et al. (2017). Tissues were collected for experiments 3-5d after surgery (Extended Data Fig. 2-1). This time period was chosen to maximize recovery time from the initial injection without allowing the animals to experience long-term discomfort or death because of the resulting neurodegeneration. Throughout the period between surgery and molecular experiments, the animals were closely monitored for weight loss and were provided with warming pads, soft food, and saline injections for hydration.

Immunofluorescence staining and imaging. Wherever possible, tissue for imaging was preserved by $4 \%$ PFA perfusion. The brains were dissected and incubated overnight at $4{ }^{\circ} \mathrm{C}$ in $4 \%$ PFA. Shortly after, they were vibratome sectioned coronally at $100 \mu \mathrm{m}$ for antibody staining and slide mounting. To visualize cSNAIL specificity (see Fig. $1 D$ ), tissues were stained with primary anti-NeuN (Cell Signaling Technology, \#12943) and secondary AlexaFluor-405 (Invitrogen, \#A-31556) to label neurons. For images related to Figure $4 D$ and Extended Data Fig. 4-4, tissues were stained with primary anti-Pvalb (Swant, PV 27) paired with secondary AlexaFluor-405 (Invitrogen, \#A-31556) or AlexaFluor-488 (Invitrogen, \#A-11034) and primary anti-Hif2a (Novus Biologicals, \#NB100-132) paired with secondary AlexaFluor-594 (Cell Signaling Technology, \#8890S). For tissues involved in both genomic assays and imaging, one fresh coronal slice, including caudate putamen, but anterior to the GPe, was fixed in 4\% PFA for $24 \mathrm{~h}$. Tissues were stained with primary anti-Tyrosine hydroxylase (TH) (Pel-Freez, \#P40101-150) and secondary AlexaFluor-647 (Invitrogen, \#A-31573) to approximate dopamine levels in the striatum. All imaging sections were mounted onto slides using ProLong Diamond Antifade mounting media (Invitrogen, \#P36961) and imaged with confocal microscopy.

Image analysis. Single-channel and colabeled cells were manually counted for Figures $1 D$ and $4 D$. To quantify the levels of striatal $\mathrm{TH}$ as a proxy for remaining dopamine (see Fig. $4 C$ ), we measured mean pixel intensity with Fiji (Schindelin et al., 2012). Measurements were performed on one $500 \times 500 \mu \mathrm{m}$ square from the striatum of each hemisphere (Extended Data Fig. 4-1), and hemisphere measurements were averaged to determine the TH levels per animal.

Nuclei collection. Fresh mouse brain tissue was sliced on a vibratome in cold, oxygenated ACSF. Then, brain ROIs were dissected under a light microscope and transferred into cold ATAC-seq lysis buffer (Buenrostro et al., 2015). The nuclei were extracted from each tissue using 30 strokes of loose pestle Dounce homogenization followed by $70 \mu \mathrm{m}$ filtration and one centrifugation wash for $10 \mathrm{~min}$ at $2000 \times g$.

Affinity purification of Sun $1 G F P^{+}$nuclei. The nuclei suspension was precleared for 10-15 min with Protein G Dynabeads (Thermo Fisher Scientific, catalog \#10004D) to remove nuclei or debris that had native affinity for the beads. Free nuclei were incubated with anti-GFP antibody (Invitrogen, \#G10362) for $30 \mathrm{~min}$ before fresh beads were added to the reaction and incubated for another $20 \mathrm{~min}$. All incubations took place at $4^{\circ} \mathrm{C}$ with $40 \mathrm{rpm}$ end-to-end rotation in wash buffer ( $0.25 \mathrm{~m}$ sucrose, 25

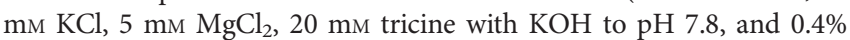
IGEPAL). After this process, the bead-bound nuclei (Sun1GFP ${ }^{+}$fraction) were purified and washed on a magnet, while the unbound nuclei were spun down and resuspended in water to preserve the Sun1GFP ${ }^{-}$ fraction.

RNA-seq library construction. After setting aside 50,000 nuclei for ATAC-seq, we extracted total RNA from all remaining nuclei in each sample using the QIAGEN RNeasy Micro Kit (catalog \#74004); 10 pg to $10 \mathrm{ng}$ of total RNA per sample was processed using the Ovation SoLo RNA-seq System with mouse AnyDeplete to remove ribosomal RNA (Tecan, catalog \#0501-32). Because many nuclear transcripts are immature, no poly A selection was performed. Libraries were paired-end sequenced at 3-9 million reads per sample on the Nextseq 500 system.

RNA-seq processing. The sequencing output files were mapped to the mm10 genome (downloaded from UCSC in November 2015) using Hisat2 with default parameters. Duplicate UMIs were removed using NuDup (https://github.com/tecangenomics/nudup). The quality of the filtered data was assessed using Picard CollectRnaSeqMetrics version 2.8.1 (http:// broadinstitute.github.io/picard). A counts per gene per sample matrix was constructed using Subread featureCounts version 1.6.4 (Liao et al., 2019) with GRCm38 gene annotations (Ensembl version 79). Sample similarities were assessed by principal component analysis (PCA) and hierarchical clustering of Euclidean sample distances after $\log 2$ transformation with 
A

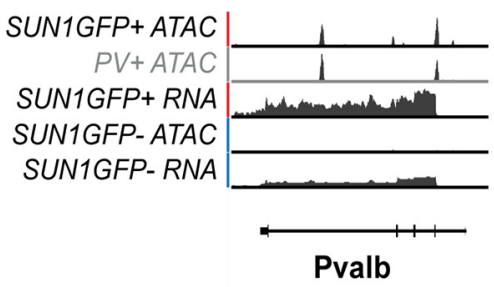

C

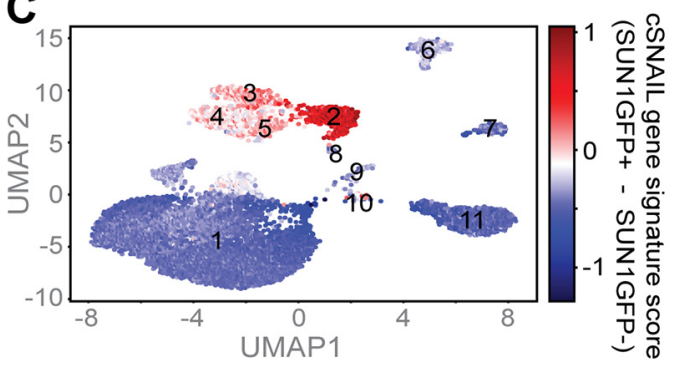

B

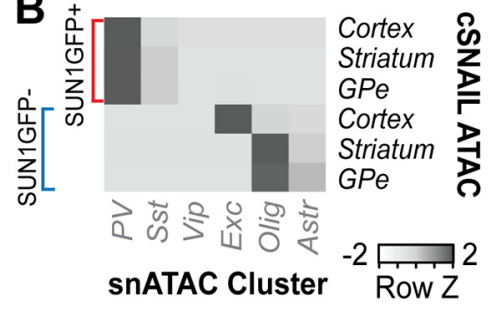

Then, samples were pooled and paired-end sequenced on a Novaseq 6000 to $\sim 25$ million unique, nonmitochondrial reads per sample for analysis.

ATAC-seq processing. The fastq files from each sample were processed with the ENCODE ATACseq pipeline (https://github.com/kundajelab/atac dnase_pipelines). Peaks were called on biological replicates of the same cell type, brain region, and treatment with an IDR threshold of 0.1 (https:// github.com/nboley/idr). This design maximizes the potential to include peaks that may only be present in one condition while limiting potential false positives that may only be called in one sample. Count tables containing the number of reads per peak for each sample were constructed using Rsubread featureCounts version 1.28.1 (Liao et al., 2019) on the filtered bam alignments. The peak sets for count tables were chosen to reflect the goals of each analysis separately. For analysis of cellular identity (see Figs. $2 B, 3 D$ ), the regions tested were the union of IDR peaks from all sham animal samples. For the promoter analysis in Figure $3 E$, instead of ATAC-seq peaks, counts were computed within $10 \mathrm{~kb}$ windows around the transcription start sites (TSSs) for all genes assessed in the RNA-seq analysis. To assess DD-affected regions (Fig. 5), six separate count tables were constructed for the six cell populations and represented the union of IDR peaks from sham and DD animals for the given cell type and brain region combination. Finally, to test for a relationship between GPe

DESeq2's $r \log$ () function (Love et al., 2014) (Extended Data Fig. 4-2). The RNA-seq data are available on GEO, accession number GSE157359.

Differential gene expression analysis. We conducted two primary gene expression analyses to determine (1) gene expression signatures of Sun $1 \mathrm{GFP}^{+}$and Sun $1 \mathrm{GFP}^{-}$cells in Pvalb-2A-Cre mice under normal dopamine conditions (see Fig. 3; Extended Data Fig. 3-1) and (2) expression changes in six cell populations on DD (see Fig. 4; Extended Data Fig. 4-3). Toward the first aim, differentially expressed genes between Sun $1 \mathrm{GFP}^{+}$and Sun1GFP ${ }^{-}$cells in each brain region were assessed in 2 sham animals. Here, we used the negative binomial model in DESeq2 version 1.14.1 (Love et al., 2014): read count $=\sim$ sex + Sun1GFP status. For the second analysis, we compared the expression profiles of each cell population between DD and sham mice. Because transcript levels are quantitative, we modeled the RNA-seq counts continuously according to the mean anti-TH fluorescence intensities of each animal, which reflect the extent of DD: DESeq2 read count $=\sim \operatorname{sex}+\mathrm{TH}$ intensity. Throughout all analyses, each model was built individually and only included the samples under direct pairwise comparison, ensuring that dispersion estimates were restricted to the relevant samples. We assessed annotation enrichments in differential gene of interest sets using g: Profiler with default parameters (see Fig. 3C; Extended Data Figs. 3-4, 45) (Raudvere et al., 2019).

ATAC-seq library construction. A small subset of nuclei was stained with DAPI, and fluorescent nuclei were manually counted using a hemocytometer to determine their concentration; $\sim 50,000$ nuclei were transferred to a new tube in $22.5 \mu \mathrm{l}$ water and mixed with $2.5 \mu \mathrm{l}$ Tagment DNA Enzyme I and $25 \mu$ l Tagment DNA Buffer (Illumina, catalog \#20034198). The tn 5 transposition reaction was conducted at $37^{\circ} \mathrm{C}$ for $30 \mathrm{~min}$ with $300 \mathrm{rpm}$ mixing. After tagmentation, the samples were taken through column purification, amplification, and double-sided size selection (100-1000 bp) with AmpureXP beads (Beckman Coulter, catalog \#A63881). To reduce amplification bias, we evaluated a side qPCR reaction for each sample to determine the optimal number of PCR cycles for each sample to reach one-third maximum intensity. To get initial sample quality estimates and balance sample representation for deep sequencing, each sample was sequenced at 1-3 million reads on the Illumina Miseq system. $\mathrm{PV}^{+}$promoter accessibility and transcription in DD (Extended Data Fig. 5-6), we derived a count table for all GPe $\mathrm{PV}^{+}$ATAC-seq samples within $2 \mathrm{~kb}$ windows around each TSS. Sample similarities were assessed among all ATAC-seq samples by PCA and hierarchical clustering using DESeq2 on a count table across the merged union of peaks from all samples (Extended Data Fig. 5-1A,B). Additionally, we ensured that each sample had TSS signal enrichment and exhibited the characteristic fragment length periodicity of high-quality ATAC-seq data, reflective of nucleosome positioning (Extended Data Fig. 5-1C,D). The ATAC-seq data are available on GEO, accession number GSE157359.

Differential open chromatin analysis. In parallel to the RNA-seq analysis, we assessed differentially accessible chromatin regions between Sun $1 \mathrm{GFP}^{+}$and Sun $1 \mathrm{GFP}^{-}$fractions in sham animal samples and regions that were affected by DD in each cell type. Sun $1 \mathrm{GFP}^{+}$and Sun1GFP comparisons in each brain region were assessed in DESeq2 version 1.14.1 (Love et al., 2014) with read count $=\sim$ sex + Sun1GFP status (see Figs. 2B, 3D,E; Extended Data Figs. 3-2, 3-3). For DD-affected open chromatin analyses (see Fig. 5; Extended Data Figs. 5-2, 5-6), we used a binary model of treatment state, DD versus saline, controlling for sex differences: read count $=\sim$ sex + treatment. Again, models for each cell population were built separately and only included samples for one comparison. Differential ATAC-seq peak sets of interest were subjected to pathway enrichment analysis using GREAT version 3.0.0 (Extended Data Figs. 5-4, 5-5) (McLean et al., 2010).

Motif enrichment analysis. Differential motif enrichment analyses (see Figs. 3F, 5C; Extended Data Figs. 3-5, 5-7) were performed using AME version 5.0.5 (Mcleay and Bailey, 2010) with motifs from the nonredundant JASPAR 2018 core vertebrates database (Khan et al., 2018). First, peak sets of interest were summit-centered and unified to $500 \mathrm{bp}$. Where more than one summit was called within a peak and these summits were $>100$ bp apart, we included both entries in the analysis. For Figure $3 F$, enrichments were assessed relative to the peaks enriched in the other cell type of that tissue (e.g., cortex $\mathrm{PV}^{+}$enriched peaks were tested for motif enrichments relative to cortex $\mathrm{PV}^{-}$enriched peaks). For Figure $5 C$, enrichments were assessed relative to a background of all IDR 


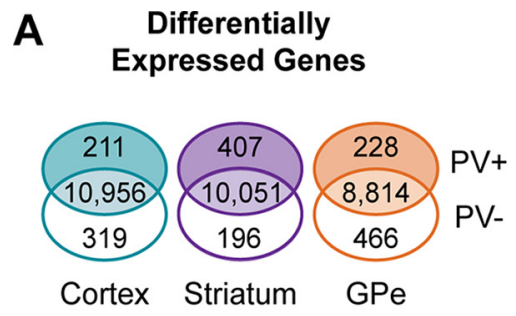

\section{Differentially Accessible Chromatin}

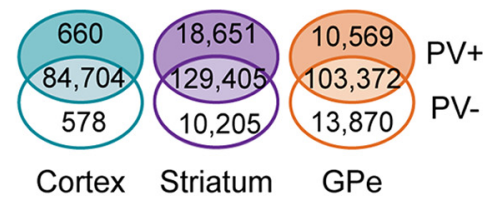

B Marker Gene Expression

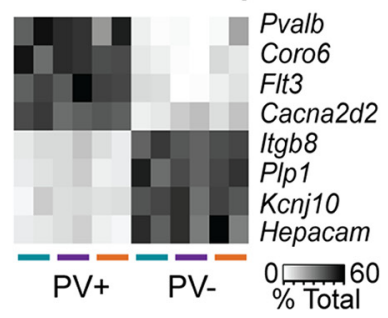

E Marker Gene Accessibility

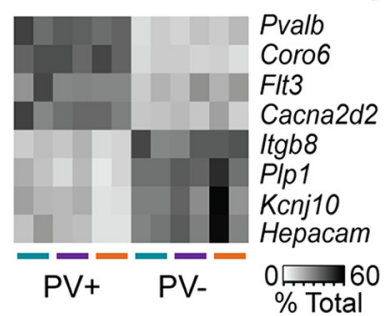

C

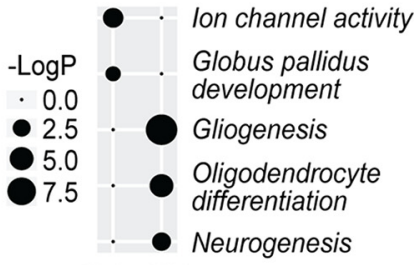

$\mathrm{PV}+\mathrm{PV}-$

F
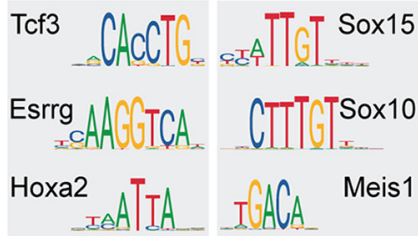

$\mathrm{PV}+\quad \mathrm{PV}-$

Figure 3. Molecular signatures of $\mathrm{PV}^{+}$neurons across brain regions. $\boldsymbol{A}$, Numbers of differentially expressed genes $\left(p_{\text {adj }}<0.01\right.$ and $\| \log 2$ FoldDifference $\left.>1\right)$ between CSNAlL-isolated PV ${ }^{+}$ and PV' fractions from the cortex, striatum, and GPe of sham animals (for details, see Extended Data Figure 3-1). B, Normalized read counts from example marker transcripts in each sample. DESeq2 normalized counts were converted to the proportion that they represent of the total read count for that gene within the given brain region. C, Example pathway enrichments for pan$\mathrm{PV}^{+}$and pan-PV ${ }^{-}$marker genes (for the full list, see Extended Data Figure 3-4). The size of the bubble represents the negative log of the adjusted $p$ value after multiple hypotheses correction. $D$, Numbers of cell type-enriched ATAC-seq peaks ( $p_{\text {adj }}<0.01$ and $\mid \log 2 F$ FoldDifference| $>1$ ) in PV ${ }^{+}$populations from each brain region (Extended Data Figure 3-2). E, DESeq2 normalized counts of ATAC-seq reads within $5 \mathrm{~kb}$ of the TSS of marker genes. Again, counts are represented as the proportion of total counts for that TSS region in the cortex, striatum, or GPe. For the full list of differentially accessible promoters, see Extended Data Figure 3-3. $\boldsymbol{F}$, Examples of motifs enriched in PV ${ }^{+}$specific or PV specific ATAC-seq peaks (Extended Data Figure 3-5). Each brain region was assessed separately, and these motifs were significantly enriched in $\mathrm{PV}^{+}$or $\mathrm{PV}^{-}$sequences across all brain regions.

peaks called in cells of the same brain region and Sun1GFP fraction as the foreground.

Single nucleus (sn)ATAC-seq processing. To compare cSNAIL-isolated cell types with single nucleus sequencing cluster markers, we reprocessed a publicly available snATAC-seq dataset from adult mouse cortex, provided by $10 \times$ Genomics. The data were downloaded and processed following the Signac adult mouse brain vignette (https:// github.com/timoast/signac). We defined peaks that marked each cluster as those with $\log 2$ FoldDifference $>0.25$ and $p<0.05$ relative to all other clusters.

snRNA-seq processing. Raw count matrices derived from $10 \times$ chromium v3 experiments conducted in mouse primary motor cortex were downloaded from NEMO (https://portal.nemoarchive. org, samples PBICCNSMMRMOPI70470511BD180328, PBICCNSMMRMOPI7047059TD180328, PBICCNSMMRMOPI70470512BD180328B, PBICCNSMMRMOPI70470512BD180328C, PBICCNS MMRMOPI70470510TD180328, and PBICCNSMMRMOPI70470512BD180328A). Empty droplets were removed using the DropletUtils package (Griffiths et al., 2018) defaultDrops method. Doublets were predicted and removed using the SCDS package (Bais and Kostka, 2019) cxds_bcds_hybrid method with a score cutoff of 1.0. The UMI count values were normalized using pooled size factor normalization (Lun et al., 2016a) implemented in the Scran package (Lun et al., 2016b). Size factor normalization requires a preclustering step, which was computed using the Scanpy (Wolf et al., 2018) package's Leiden community detection algorithm (Traag et al., 2019). To create the UMAP (McInnes et al., 2018) for visualization, we identified the top 7500 most variable genes using scanpy. pp.highly_variable_genes with flavor "seurat" and computed the principle components on these genes with scanpy.pp.pca. The UMAP was then computed based on the PCA reduced single nucleus gene expression matrix. To compute a PV gene signature score for each cell, we averaged the $\mathrm{PV}^{+}$positively differentially expressed genes in the scran normalized, mean centered, and SD scaled gene expression matrices and subtracted the averaged $\mathrm{PV}^{-}$positively differentially expressed genes in the same matrices.

Statistical analyses. To determine the specificity and efficiency of cSNAIL labeling in each brain region (Fig. 1E), these metrics were quantified for four independent images and averaged. This approach is more appropriate than computing the specificity on the pool of all cells together because treating images as replicates allows us to also quantify variation in the data. To this end, the SEM across the four images is reported for each brain region. The same principles were used for the quantification of TH (Extended Data Fig. 4-1) and Hif2 $\mathrm{a}^{+} \mathrm{PV}^{+}$neurons on DD (Fig. 4D). Images from tissue of the same treatment state (DD or no DD) were treated as replicates and the spread of the data are shown by the box plot. To test for a difference between the means of the two treatment groups, we used two-tailed $t$ test statistics.

For comparing cSNAIL ATAC-seq markers to snATAC-seq cluster markers (Fig. 2B), we used the one-sided hypergeometric enrichment test, sampling cell type-specific cSNAIL peaks in each brain region from an expected population frequency of cluster-specific snATAC-seq peaks. The hypergeometric distribution is commonly used to test for association of binary variables in genomics and is suitable to help us interrogate which clusters have the most in common with cSNAIL-isolated cells. Only peaks present in both the cSNAIL-isolated dataset and the snATAC-seq dataset $(N=112,082)$ were evaluated. For simplicity, cluster-specific peaks from all excitatory neuron clusters were combined into one category. For visualization, we converted the hypergeometric test enrichments throughout each cSNAIL cell population into $z$ scores and plotted them using gplots heatmap.2 in R (https:/github.com/ talgalili/gplots).

In order to visualize the extent to which each snRNA-seq nucleus had a similar expression profile to cSNAIL-isolated nuclei (Fig. 2C), we computed a per-nucleus gene signature score that is the difference between the normalized expression of Sun $\mathrm{GFP}^{+}$specific genes and Sun1GFP ${ }^{-}$specific genes. Because the snRNA-seq came from the healthy cortical tissue, we focused on genes that were differentially expressed between sham cortical Sun $1 \mathrm{GFP}^{+}$and Sun $1 \mathrm{GFP}^{-}$fractions. The Sun $1 \mathrm{GFP}^{+}$and Sun1GFP ${ }^{-}$scores were computed by averaging across the scaled ( 0 mean centered, unit variance) expression of signature genes across all cells.

For differential gene expression and differential chromatin accessibility analyses (see Figs. 3-5), genes or ATAC-seq regions were assessed using the negative binomial model in DESeq2 version 1.14 .1 with Wald test statistics. The parameters and inputs of models were tailored to each 
A

\begin{tabular}{|c|c|c|c|c|c|c|}
\cline { 2 - 7 } \multicolumn{1}{c|}{ RNA-seq } & Cortex PV+ & Cortex PV- & Striatum PV+ & Striatum PV- & GPe PV+ & GPe PV- \\
\hline Genes Tested & 26245 & 26860 & 25149 & 24737 & 17430 & 26325 \\
\hline$\uparrow$ in DD & 0 & 0 & 0 & 0 & 14 & 0 \\
\hline$\downarrow$ in DD & 0 & 3 & 0 & 0 & 15 & 3 \\
\hline
\end{tabular}

B

DD-Affected Genes in GPe PV+ neurons

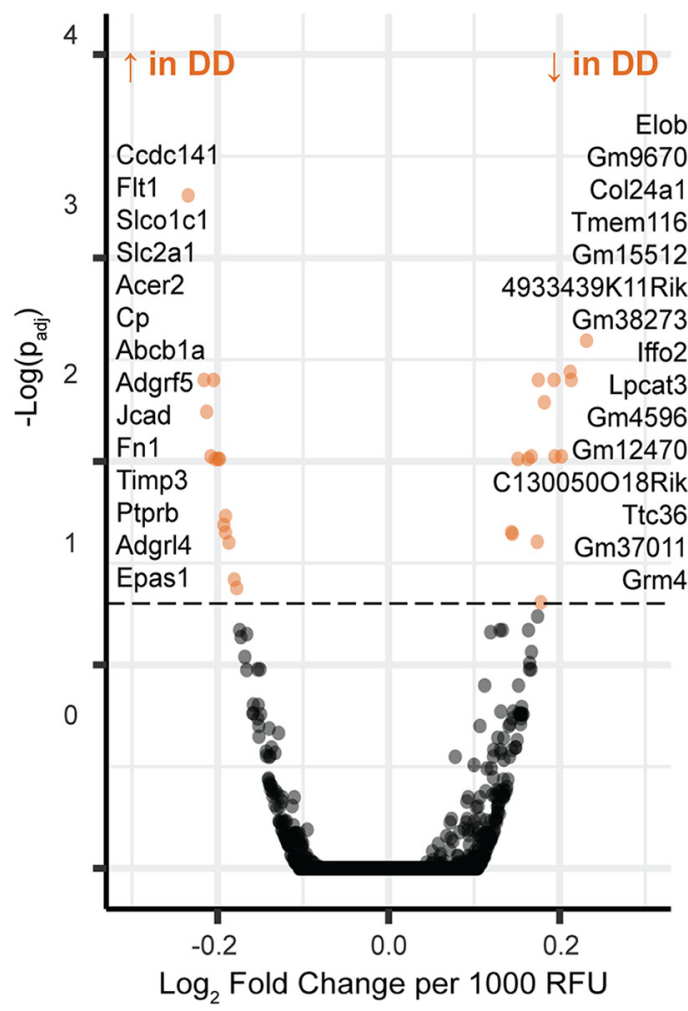

C HIF pathway gene expression

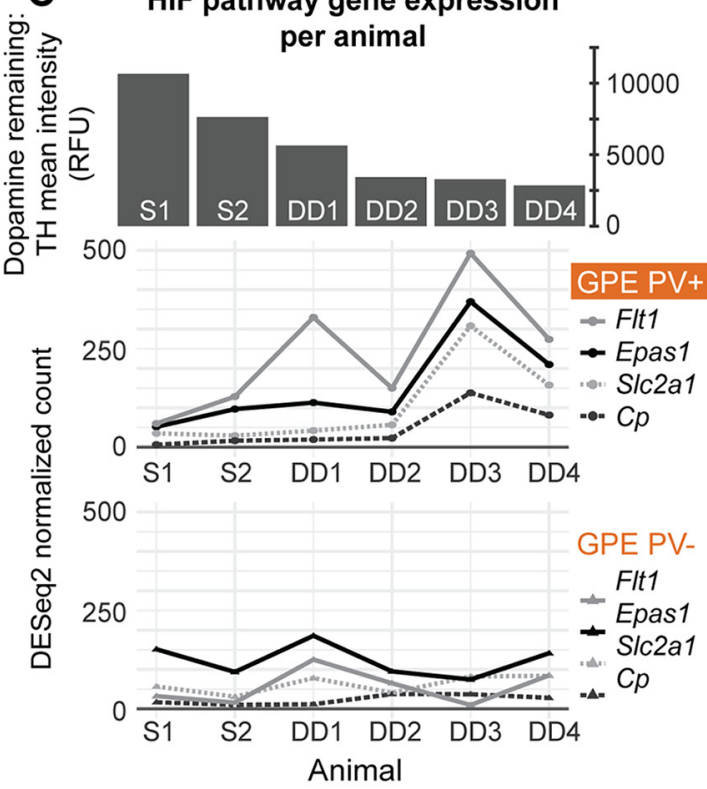

D Hif2a immunopositive GPe PV+ neurons

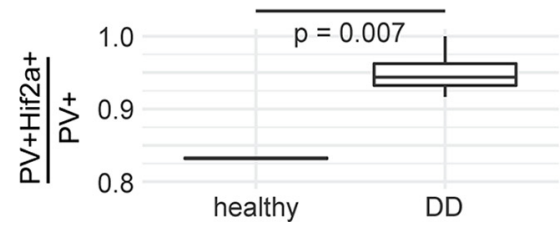

Figure 4. Divergent gene expression in dopamine-depleted mice is largely restricted to GPe PV ${ }^{+}$neurons and implicates the HIF pathway. $A$, Numbers of genes that have differential expression with DD in each cell type. DD was confirmed by immunochemistry (Extended Data Figure 4-1), and RNA-seq sample quality was confirmed computationally (Extended Data Figure 4-2). $\boldsymbol{B}$, Volcano plot of DD-affected genes in GPe PV ${ }^{+}$neurons. Orange represents genes meeting the significance threshold of $p_{\text {adj }}<0.05$, listed in order of significance. See Extended Data Figure 4-3 for a detailed list of differentially expressed genes and Extended Data Figure 4-5 for functional relationships. C, Dopamine depletion is associated with a specific increase in Hif2a (Epas7) transcription and its target genes in GPe PV ${ }^{+}$neurons. The animals are referred to as $\mathrm{S1}$ and $\mathrm{S} 2$ for sham animals and DD1-4 for 6-0HDA-treated animals in order of depletion severity. TH levels are shown for each animal in the bar graph. $\boldsymbol{D}$, There is an increase in the proportion of GPe PV ${ }^{+}$cells that express Hif2a protein between images from healthy tissue and DD tissue ( $t$ test) (Extended Data Figure 4-4).

analysis (see sections above for specific model formulas), but all models included data from both sexes and controlled for sex differences. The significance of differential genes or regions was evaluated using the $\log 2$ fold difference between two cell types or conditions and the $p$ value after multiple hypothesis correction with the Benjamini Hochberg method $\left(p_{\text {adj }}\right)$. Significance cutoffs for specific analyses are described throughout Results, and the statistics for these tests can be found in Extended Data Figs. 3-1, 3-2, 3-3, 4-3, and 5-2.

To assess concordance between gene transcriptomic and epigenomic changes in GPe $\mathrm{PV}^{+}$neurons on DD (Extended Data Fig. 5-6), we categorized genes into nonaffected, DD-increasing, or DD-decreasing and plotted the $\log 2$ fold difference of ATAC-seq signal within a $2 \mathrm{~kb}$ window around the TSS of these genes. We tested for a difference in population means between these groups using the two-tailed $t$ test statistics.

Gene annotation enrichment analyses are a similar statistical problem as the snATAC-seq cluster marker .comparison in that the objective is to test for overrepresentation of features belonging to a certain category within a feature set of interest. Therefore, we also used the hypergeometric test in this instance, as implemented by g:Profiler
(Raudvere et al., 2019) (see Fig. 3C; Extended Data Figs. 3-4, 4-5). Similarly, we used hypergeometric test statistics as implemented in GREAT version 3.0.0 (McLean et al., 2010) to explore enriched pathways associated with differential ATAC-seq peak sets of interest (Extended Data Figs. 5-4, 5-5).

Motif enrichment analyses in AME (Mcleay and Bailey, 2010) (Figs. 3F, 5C; Extended Data Figs. 3-5, 5-7) were conducted using the average odds scoring method, which calculates the average position weight matrix motif score for each input sequence, and Fisher's exact test statistics. The reported $p$ values were adjusted for multiple hypothesis testing using a Bonferroni correction.

\section{Results}

cSNAIL isolates $\mathrm{Cre}^{+}$nuclei with high precision

cSNAIL technology is a viral strategy for labeling and isolating the nuclei of Cre-expressing cells. To achieve nuclei isolation compatible with epigenomic profiling, we used a similar strategy 
A

\begin{tabular}{|c|c|c|c|c|c|c|}
\cline { 2 - 7 } \multicolumn{1}{c|}{ ATAC-seq } & Cortex PV+ & Cortex PV- & Striatum PV+ & Striatum PV- & GPe PV+ & GPe PV- \\
\hline Regions Tested & 118733 & 127730 & 108126 & 125257 & 95235 & 112529 \\
\hline$\uparrow$ in DD & 0 & 2361 & 26 & 3075 & 16 & 403 \\
\hline$\downarrow$ in DD & 1 & 2483 & 15 & 3622 & 18 & 431 \\
\hline
\end{tabular}

B
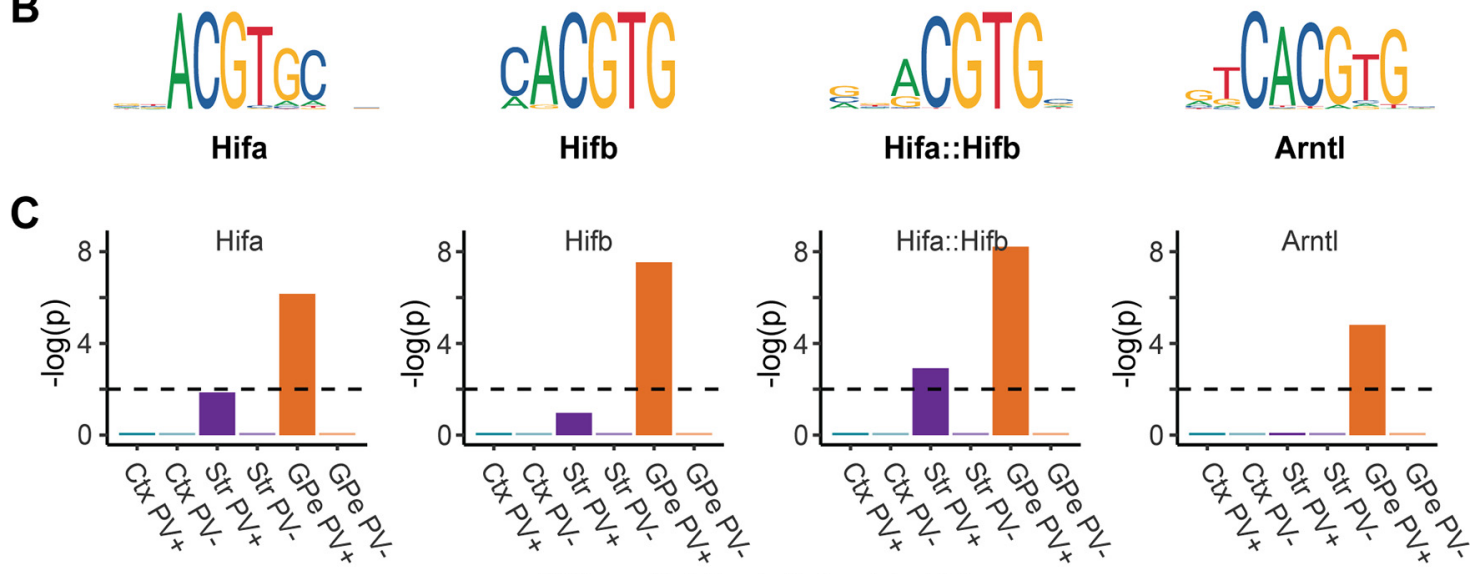

Differential Peak Sets: $\uparrow$ in DD

Figure 5. Open chromatin changes associated with DD show cell type-specific HIF motif enrichment. $\boldsymbol{A}$, Numbers of ATAC-seq regions in each cell type that are differentially open in DD animals compared with sham animals. The data input into these models were high quality (Extended Data Figure 5-1). Full descriptions of differentially accessible regions and potential functions can be found in Extended Data Figures 5-2, 5-3, 5-4, and 5-5. The relationship between TSS accessibility and gene expression in GPe PV ${ }^{+}$neurons is shown in Extended Data Figure 5-6. $\boldsymbol{B}$, HIF family transcription factor binding motifs as defined in JASPAR 2018 (Khan et al., 2018). C, Cell type-specific HIF family motif enrichments among sets of sequences underlying ATAC-seq peak summits that increase in accessibility in DD animals. The full set of enriched motifs are available in Extended Data Figure 5-7.

to the Sun1GFP INTACT transgenic mouse strain (Mo et al., 2015). Sun1 is a highly conserved nuclear envelope protein that helps connect the nucleoskeleton and the cytoskeleton (Haque et al., 2010). Because of its tight association with the inner nuclear envelope, the Sun1GFP fusion protein localizes the GFP protein to the nuclear surface. This positioning allows for the affinity purification of nuclei, whereby after tissue is homogenized into a single-nuclei suspension, magnetic beads coated with anti-GFP antibody specifically bind Sun1GFP ${ }^{+}$nuclei, and they can be separated from Sun $1 \mathrm{GFP}^{-}$nuclei with a magnet. Faced with the small capacity of AAV, we made several modifications to the INTACT mouse transgene design (see Materials and Methods). The resulting cSNAIL genome expresses a modified Sun1GFP protein in a Cre-dependent manner. We packaged the cSNAIL Sun1GFP vector into AAV variant PHP.eB, which is capable of crossing the blood-brain barrier to broadly transduce the CNS (Chan et al., 2017).

We evaluated the ability of cSNAIL to drive Cre-specific nuclear anchored independent labeling in the Pvalb-2A-Cre mouse line with imaging and molecular data (Figs. 1,2). With fluorescent imaging, we confirmed that cSNAIL promotes Sun1GFP expression that is properly localized to the nuclear envelope in tissue (Fig. 1B). This expression is sufficient to bind anti-GFP coated magnetic beads after tissue dissociation (Fig. 1C). To quantify the ability of cSNAIL to correctly target $\mathrm{Cre}^{+}$cells, we compared the expression of cSNAIL to the expression of Cre reporter tdTomato of the Pvalb-2A-Cre/Ai14 mouse strain (Fig. $1 D, E)$. For the purposes of this analysis, we defined virus specificity as the percent of Sun $1 \mathrm{GFP}^{+}$cells that also express tdTomato and virus efficiency as the percent of tdTomato ${ }^{+}$cells that also express Sun1GFP. cSNAIL exhibited very strong (>94\%) specificity in the cortex, striatum, and GPe. The efficiency ranged from $53 \%$ to $81 \%$, which reflects the expected transduction of the viral capsid in these brain regions (Chan et al., 2017).

To confirm the molecular identity of cSNAIL-isolated nuclei from Pvalb-2A-Cre mice, we compared ATAC-seq and RNA-seq from sham animal Sun $1 \mathrm{GFP}^{+}$and Sun $1 \mathrm{GFP}^{-}$fractions to publicly available $\mathrm{PV}^{+}$and $\mathrm{PV}^{-}$data. cSNAIL ATAC-seq and RNAseq exhibited the expected cell type-specific patterns at the Pvalb locus compared with signals from INTACT-isolated populations (Mo et al., 2015) (Fig. 2A). When comparing our cell type-specific ATAC-seq markers to single nucleus (sn)ATAC-seq clusters from the adult mouse cortex (https://github.com/timoast/signac), the Sun $1 \mathrm{GFP}^{+}$peaks from each brain region were enriched for $\mathrm{PV}^{+}$cluster markers (Fig. 2B). Aligning with the expected cell type proportions in each brain region, Sun $1 \mathrm{GFP}^{-}$fractions were most enriched for snATAC-seq markers of excitatory neuron clusters (cortex only) and glial clusters.

We focused on marker open chromatin peaks from clusters of cells because of the sparse, generally binary nature of snATAC-seq counts. On the other hand, the quantitative properties of snRNA-seq transcript counts enable a richer interpretation of cSNAIL signatures at the level of individual nuclei. Taking advantage of this, we explored which snRNA-seq nuclei from the mouse primary motor cortex had similar expression patterns to our cortical cSNAIL-isolated populations. First, we defined cSNAIL Sun $1 \mathrm{GFP}^{+}$and cSNAIL Sun1GFP ${ }^{-}$gene expression signatures in the cortex (i.e., strongly differentially expressed genes) (Extended Data Fig. 3-1). Then we scored snRNA-seq nuclei based on the difference between their expression of the cSNAIL Sun $1 \mathrm{GFP}^{+}$and cSNAIL Sun $1 \mathrm{GFP}^{-}$gene signatures (Fig. 2C; Extended Data Fig. 2-2). As expected, nuclei in the $\mathrm{PV}^{+}$neuron cluster had the strongest expression of the 
cSNAIL Sun $1 \mathrm{GFP}^{+}$gene signature, followed by other inhibitory neuron clusters. All excitatory neuron and non-neuronal clusters tended to contain cells with dominating cSNAIL Sun1GFP ${ }^{-}$ signatures. Together, these comparisons provide evidence that cSNAIL Sun $1 \mathrm{GFP}^{+}$samples are indeed strongly enriched for $\mathrm{PV}^{+}$ neurons. Therefore, throughout the remainder of this paper, we refer to cSNAIL Sun $1 \mathrm{GFP}^{+}$and Sun1GFP ${ }^{-}$cells as $\mathrm{PV}^{+}$and $\mathrm{PV}^{-}$ cells, respectively.

Molecular signatures of cSNAIL-isolated $\mathrm{PV}^{+}$and $\mathrm{PV}^{-}$cells In each brain region, we recovered hundreds of differentially expressed genes and thousands of differentially accessible ATAC-seq regions between $\mathrm{PV}^{+}$and $\mathrm{PV}^{-}$cell populations (Fig. 3A,D; Extended Data Figs. 3-1, 3-2). We defined 35 $\mathrm{PV}^{+}$and $29 \mathrm{PV}^{-}$marker genes with high specificity (DESeq2 $p_{\text {adj }}<0.01$ and $\mid \log 2$ FoldChange $\mid>1$ ) across all three tissues. Of these genes, a small number also had corresponding cell typespecific chromatin accessibility within a $10 \mathrm{~kb}$ window around the gene TSS (Extended Data Fig. 3-3), including those highlighted in Figure $3 B, E$. Functional gene enrichment analysis on pan- $\mathrm{PV}^{+}$and $\mathrm{PV}^{-}$differential genes implicated neuron-related pathways among the $\mathrm{PV}^{+}$signature and glial pathways, especially oligodendrocyte-related pathways, among the $\mathrm{PV}^{-}$signature (Fig. 3C; Extended Data Fig. 3-4). This was not surprising given that neuron composition captured in $\mathrm{PV}^{-}$fractions varies greatly between the cortex, striatum, and GPe.

Next, we sought to identify transcription factor binding motifs that could play a role in epigenetically defining $\mathrm{PV}^{+}$neurons. We tested for enriched motifs in sequences underlying $\mathrm{PV}^{+}$and $\mathrm{PV}^{-}$specific ATAC-seq peaks in each brain region (Fig. 3F; Extended Data Fig. 3-5). Among the significantly enriched $\mathrm{PV}^{+}$motifs in all three brain regions was Tcef3, which has been previously implicated in $\mathrm{PV}^{+}$neuron-specific gene regulation (Mo et al., 2015). Additionally, we determined consistent $\mathrm{PV}^{+}$enrichment for the Esrrg and Hoxa2 motifs. The Esrrg transcript itself was also upregulated in $\mathrm{PV}^{+}$neurons in all three brain regions $\left(p_{\text {adj }}<0.001\right)$, but Tcef3 or Hoxa2 were not. Consistent $\mathrm{PV}^{-}$motif enrichments across all brain regions included the motif for Sox10, which is necessary for the differentiation and survival of oligodendrocytes (Pozniak et al., 2010; Takada et al., 2010). There were also significant enrichments for many other Sox family motifs and the Meis1 motif among $\mathrm{PV}^{-}$ ATAC-seq sequences (Extended Data Fig. 3-5). The representation of glial signatures among $\mathrm{PV}^{-}$cell ATAC-seq and RNA-seq indicates that our nuclei dissociation during cSNAIL affinity purification is not restricted to neurons.

\section{Gene expression differences in dopamine-depleted animals}

Severe DD was induced with bilateral acute 6-OHDA lesions, while control animals received a sham surgery of a saline injection under the same conditions. Molecular experiments were conducted 3-5d after surgery to precisely replicate the timing when $\mathrm{GPe} \mathrm{PV}^{+}$neuron stimulation is behaviorally significant (Mastro et al., 2017). All DD experiments were conducted when the mice were heavily symptomatic, before reaching the point of death. This paradigm specifically captures the advanced rodent DD phenotype, which is physiologically similar whether the DD was acute or gradual (Willard et al., 2019). Striatal tissue from 6-OHDA-lesioned animals had significantly lower levels of dopamine production enzyme TH than sham animals, indicating successful depletions (Extended Data Fig. 4-1). All depletions were bilateral, except in 1 animal, DD2, where one hemisphere was only weakly depleted. The RNA-seq data were high quality for all samples except one striatum $\mathrm{PV}^{-}$sample from a $\mathrm{DD}$ animal, which was excluded from the analysis. Samples separated by tissue and cell type in PCA and hierarchical clustering, indicating higher variability between cell types than between biological replicates (Extended Data Fig. 4-2).

In the RNA-seq data from DD and sham animals, we recovered very few significant DD-affected genes in any cortical cell populations, striatal cell populations, or in the GPe $\mathrm{PV}^{-}$population. In contrast, $\mathrm{GPe} \mathrm{PV}^{+}$neurons contained 29 differentially expressed genes in DD ( $p_{\text {adj }}<0.05$ ) (Fig. $4 A, B$; Extended Data Fig. 4-3). It was readily apparent that there were many genes involved in cellular oxygen homeostasis and neuroprotection among the 14 genes that were upregulated in the $\mathrm{GPe} \mathrm{PV}^{+}$neurons of DD animals. These included Epas1, which encodes for Hypoxia-inducible factor $2 \alpha$ (transcription factor Hif2a), and three of its targets: Cp, Slc2a1, and Flt1 (Schofield and Ratcliffe, 2004; Dengler et al., 2014; Smeyne et al., 2015). Additionally, the downregulated set included Elob, a moderator of free oxygen sensing that inhibits Hif2a activity (Ohh et al., 2000). These results suggest that $\mathrm{GPe} \mathrm{PV}^{+}$neurons may react to DD through hypoxia-inducible factor (HIF) signaling and associated transcriptional responses.

To see whether increased Epas1 transcription in $\mathrm{GPe} \mathrm{PV}^{+}$ neurons was accompanied by increased Hif2a protein levels, we performed double immunofluorescent staining for Pvalb and Hif2a in healthy and DD mouse tissue. Indeed, a higher proportion of GPe $\mathrm{PV}^{+}$neurons expressed Hif2a in images from DD animals compared with healthy animals (Fig. 4D; Extended Data Fig. 4-4). The change in mean proportion was $11.3 \%$, and this difference was significant ( $t$ test, $p=0.007$ ).

Other differentially expressed genes in the $\mathrm{GPe}^{+} V^{+}$neurons of DD mice have also been implicated in processes of neurodegeneration and neuroprotection. For example, we observed overexpression of Timp3, a key inhibitor of matrix metalloproteinases which contribute to dopaminergic neuron apoptosis in PD (Kim et al., 2007). Timp3 expression in neurons is protective against blood-brain barrier damage, but high levels of Timp3 can lead to cell death (Rosenberg, 2009). Lpcat3, a gene we found to have reduced expression in $\mathrm{GPe} \mathrm{PV}^{+}$neurons on DD, is necessary for ferroptosis, which has been linked to neurodegeneration in $\mathrm{PD}$ and Alzheimer's disease (Stockwell et al., 2017). Because of the small number of regulated genes, functional enrichment for specific pathways was low (Extended Data Fig. 4-5).

\section{Open chromatin differences in dopamine-depleted animals}

In addition to transcriptome profiling, the biology of epigenetics suggests that it could provide valuable information about the transcriptional network activated during DD. In contrast to gene expression, the epigenetic state of a cell can be more reflective of its past history as well as its future potential to respond to a stimulus (West et al., 2001; Day and Sweatt, 2011). For example, epithelial stem cells retain a "memory" of acute stress in their chromatin, which later influences the genes that are expressed in response to a secondary challenge (Naik et al., 2017). This is also true in the brain, where the epigenetic state of a brain region, and even specific cell types, is highly correlated with how a gene responds to neural activity (Spiegel et al., 2014; Whitney et al., 2014). DNA methylation and histone modification differences have been described in PD, mostly in blood and the SNpc, suggesting that epigenetic gene regulation may be important in this context (van Heesbeen and Smidt, 2019). In this study, we compared the chromatin landscapes between DD and sham animals in each cell population with ATAC-seq, a measurement that is 
correlated with cis regulatory element activity (Buenrostro et al., 2013).

Based on standard QC metrics (see Materials and Methods), the ATAC-seq data were high quality for all samples, except one cortex $\mathrm{PV}^{+}$sample from a DD animal, which was excluded from the analysis (Extended Data Fig. 5-1). In DD animals, we observed a small number of confident open chromatin changes in $\mathrm{PV}^{+}$cell types and many changes within $\mathrm{PV}^{-}$cell types that met significance at a cutoff of $p_{\text {adj }}<0.05$ (Fig. 5A; Extended Data Fig. 5-2). The DDaffected open chromatin regions in different cell types had some redundancy, but the majority were cell type-specific at the level of the individual regulatory element (Extended Data Fig. 5-3) (Gu et al., 2014). However, DD-affected regions in multiple cell types tended to converge around areas of the genome enriched for certain functions, including neurotransmission, immune response, and the methionine cycle (Extended Data Figs. 5-4, 5-5).

We then assessed whether the observed differences in open chromatin could be responsible for the transcriptional differences observed in the $\mathrm{GPe}^{+} \mathrm{PV}^{+}$population (Extended Data Fig. 5-6). Genes with increased expression in DD on average had higher levels of open chromatin at their promoters, but the shift in means was not significant. As a population, genes that decreased in expression on DD had no DD-associated shift in promoter accessibility relative to nonaffected genes. Additionally, there were no overlaps in the most significantly differential genes and peaks. These findings suggest that the mechanisms of gene regulation in this case may favor distal cis interactions and trans factors.

Because of the increased expression of HIF-related transcripts in $\mathrm{GPe} \mathrm{PV}^{+}$neurons in $\mathrm{DD}$, we hypothesized that changing open chromatin regions in this cell type would contain HIF transcription factor binding motif sites. Upon activation, Hifa subunits heterodimerize with Hifb and the complex binds regulatory elements in the genome to affect transcription. Therefore, we conducted a motif enrichment analysis for four HIF family motifs with Fisher's exact test statistics. These included the JASPAR 2018 motifs (Khan et al., 2018) for (1) Hifla (MA1106.1), which has core motif redundancy with Hif2a (Schödel et al., 2011), (2) Arnt or Hiflb (MA0004.1), (3) the Hifa-Hifb complex (MA0259.1), and (4) a related Hifa binding partner Arntl (M0603.1) (Fig. 5B). Because there were too few examples of DD-affected $\mathrm{PV}^{+}$peaks for statistical enrichment analysis at this threshold, we assessed $\mathrm{PV}^{+}$enrichments within peaks that met a more lenient threshold of $p_{\text {adj }}<0.2$ (Extended Data Fig. 5-2). As predicted, all four motifs were highly enriched within $\mathrm{GPe} \mathrm{PV}^{+} \mathrm{DD}$-increasing peaks (i.e., the set of ATAC-seq peaks with increased accessibility in on DD) (Fig. 5C). Moreover, the motifs were not enriched in DD-increasing peak sets from other cell types with the exception of some weaker enrichment in striatal $\mathrm{PV}^{+}$neurons. Finally, sets of ATAC-seq peaks with decreased accessibility in DD (DD-decreasing) were not enriched for HIF motifs in any cell type, including $\mathrm{GPe} \mathrm{PV}^{+}$. These results indicate a cell type-specific induction of $\mathrm{HIF}$ signaling in $\mathrm{GPe} \mathrm{PV}^{+}$neurons on DD.

To assess which additional transcription factors may be involved in the epigenetic response to DD, we extended the analysis to all motifs within the nonredundant JASPAR 2018 core vertebrates database (Khan et al., 2018) (Extended Data Fig. 5-7). Specific to DD-increasing $\mathrm{GPe} \mathrm{PV}^{+}$peaks and, to a lesser extent, also DD-increasing striatal $\mathrm{PV}^{+}$peaks, we observed an enrichment for Ets-related transcription factor motifs, including Gabpa $\left(\mathrm{GPe} p_{\text {adj }}=1.25 \mathrm{e}-30\right.$, striatum $\left.p_{\text {adj }}=1.41 \mathrm{e}-10\right)$. Gabpa is an essential regulator of many cellular respiration genes, including multiple cytochrome $c$ oxidase subunits (Ongwijitwat et al., 2006).
DD-affected open chromatin regions in other cell types contained enrichments for several additional transcription factor binding motifs. Notably, DD-increasing peak sets in every cell population, except for $\mathrm{GPe} \mathrm{PV}^{+}$, were all enriched for the binding motifs of glucocorticoid receptor transcription factors $\mathrm{Nr} 3 \mathrm{cl}$, $\mathrm{Nr} 3 \mathrm{c} 2$, and $\mathrm{Ar}\left(p_{\mathrm{adj}}<0.01\right)$. Glucocorticoid receptors are emerging as key regulators of neuroinflammation, and disruption of their proper regulation is thought to play a role in the onset of $\mathrm{PD}$ by allowing the infiltration of cytotoxins in the $\mathrm{SNpc}$ (Herrero et al., 2015). Our findings are consistent with similar stressors in the cortex, striatum, and GPe.

\section{Discussion}

We began with a query on the cell type-specific molecular changes in $\mathrm{GPe} \mathrm{PV}^{+}$neurons of 6-OHDA-treated DD mice. cSNAIL technology enabled the efficient isolation of this precise cell population and the others in these experiments. Because cSNAIL is delivered through AAV, we were able to conduct the molecular experiments on single transgenic animals instead of double transgenics, reducing animal use. Furthermore, cSNAIL packaging in the PHP.eB capsid enabled whole-brain transduction on intravenous injection, maximizing cell yield from each individual and minimizing the confounding inflammation associated with direct injection to the brain. The cSNAIL labeling and isolation system is immediately compatible with most available Cre transgenic strains.

Compared with other technologies, nuclear surface labeling strategies, such as INTACT, are uniquely well suited for the isolation of rare cell types for genomic and epigenetic assays. cSNAIL marks an important advance in cell isolation because it evolves the advantages of immunopurification tagging into a system that is much more flexible and scalable. The Sun1GFP peptide design in cSNAIL is the first demonstration of a nuclear surface tag gene that is compact enough to be packaged in AAV. This Sun1GFP variant can be easily incorporated into other constructs to achieve unprecedented access to small cell populations in a strain-independent manner. For example, the Sun1GFP gene could be expressed under the control of a cell type, region, or condition-specific regulatory element (Blankvoort et al., 2018). Unlike the generation of new transgenic lines, new cSNAIL adaptations can be simply created through vector cloning and have the potential to be transferrable across species. In the future, cSNAIL may also be paired with snRNA-seq and snATAC-seq to conduct more detailed profiling of molecular diversity within a genetically defined cell population.

Using cSNAIL technology in Pvalb-2A-Cre mice, we characterized the defining transcriptional and epigenomic features of $\mathrm{PV}^{+}$neurons across the cortex, striatum, and GPe. It is important to note that this implementation of cell sorting with cSNAIL was Cre-dependent. Thus, these populations directly reflect the cells captured in this Cre mouse strain, which may differ from other definitions of $\mathrm{PV}^{+}$neurons. Additionally, because we compared with $\mathrm{PV}^{-}$cell population data and not other isolated subtypes, some $\mathrm{PV}^{+}$enriched signatures may be general features of inhibitory neurons and not necessarily restricted to $\mathrm{PV}^{+}$ neurons.

Consistent with previous electrophysiological observations and interventions, we found that $\mathrm{GPe} \mathrm{PV}^{+}$neurons were a critically affected population in the DD brain. Indeed, in this paradigm, $\mathrm{GPe} \mathrm{PV}^{+}$neurons were the only cell population with notable gene expression changes after DD out of the six included cell populations. This is in contrast to some previous reports 
describing transcriptional alterations in the striatum in PD brain tissue (Miller et al., 2006; Botta-or et al., 2012). While acute animal models are not sufficient to capture the complexities of human PD, they are valuable for understanding some specific consequences of dopamine depletion in a direct manner. Certain other animal models of DD, including MPTP lesion and levodopa-induced models, also produce striatal transcription changes (Chin et al., 2008; Heiman et al., 2014). In this case, we selected the specific paradigm we used to best answer our hypothesis about $\mathrm{GPe} \mathrm{PV}^{+}$neurons in late-stage DD. We acknowledge that other animal models may be better suited for striatum-specific inquiries or to examine timing-specific effects throughout the progression of $\mathrm{PD}$. In this severe, acute model of $\mathrm{DD}$, we found evidence that the HIF signaling pathway, part of the cellular oxidative stress response, is induced in $\mathrm{GPe} \mathrm{PV}^{+}$neurons. These results implicate new molecular changes within the neural circuit for movement production, but outside the SNpc, as a consequence of DD.

Oxidative stress is known to be an important factor for the degeneration of dopaminergic neurons during the onset of PD and is thought to be a consequence of mitochondrial dysfunction. Postmortem SNpc tissue from idiopathic PD patients contains deficits in mitochondrial complex I of the electron transport chain (Schapira et al., 1990). This is also the specific deficit caused by the MPTP animal model of PD (Langston et al., 1984; Heikkila et al., 1989; Schapira et al., 1990). Other cellular and animal models of PD indirectly compromise mitochondrial activities, including 6-OHDA induction (Blum et al., 2001; Hunter et al., 2007; Xie and Chung, 2012). Moreover, major PD risk alleles affect mitochondrial genes and genes involved in mitochondrial function, including PINK1, PRKN, DJ-1, and SNCA, among others (Larsen et al., 2018).

Previous transcriptomic assays have reported RNA abnormalities in PD related to mitochondrial dysfunction and oxidative stress in the SNpc and striatum of mice treated with MPTP, methamphetamine, or mutated $\alpha$ synuclein (Chin et al., 2008; Soreq et al., 2012). Although we did not recover transcriptomic evidence of oxidative stress in the striatum, our results support the emerging hypothesis that cellular oxygen availability influences not only the onset of PD in the SNpc, but also the downstream circuit. It is unclear whether the HIF response we observed in $\mathrm{GPe} \mathrm{PV}^{+}$neurons is a sign of cellular distress, a neuroprotective device, or both. In the SNpc, Hif2a is necessary for adult dopaminergic neuron function and is neuroprotective against dopaminergic neuron loss (Smeyne et al., 2015). There have been reports of $\mathrm{GPe} \mathrm{PV}^{+}$specific neurodegeneration in some more gradual models of DD (Hardman and Halliday, 1999; Fernández-Suárez et al., 2012), but little is known about their longevity with respect to other neurons in acute DD implementations. There may be opportunities for gene therapies involving the moderation of oxygen use in these cells, but further work is necessary to discern the potential here.

Why would transcriptional responses to DD manifest selectively in $\mathrm{GPe} \mathrm{PV}^{+}$neurons over other populations in the basal ganglia? Fast-spiking $\mathrm{PV}^{+}$neurons have high-energy demands compared with other types of neurons and are therefore more susceptible to fluctuations in oxygen availability or mitochondrial function (Kann, 2016). Disruption in cellular respiration in $\mathrm{PV}^{+}$neurons, especially during development, is an immense burden in vivo and has been linked to neuropsychiatric disorders, including schizophrenia and autism spectrum disorder (Inan et al., 2016; Steullet et al., 2017). Impaired mitochondrial function in $\mathrm{PV}^{+}$neurons is associated with changes in synaptic input onto $\mathrm{PV}^{+}$neurons, $\mathrm{PV}^{+}$neuron excitability, and network oscillations (Inan et al., 2016). Because neuronal activity itself can have drastic impacts on gene regulation (West et al., 2001), the observed transcriptional changes in GPe $\mathrm{PV}^{+}$neurons in DD could also be a consequence of changes in circuit properties.

Unlike the specific nature of the transcriptional response to $\mathrm{DD}$, the open chromatin data suggest more global epigenetic effects. These open chromatin observations could be a signature of an epigenetic "scar," where DD temporarily disrupted gene expression and an epigenomic response restored gene expression to near normal levels, but the open chromatin state remains altered (Maze et al., 2014). Under some circumstances, Hif2a has been shown to autoregulate, whereby increased Hif2a protein levels promote additional transcription of the Epas 1 gene (Sato et al., 2002). This provides a possible mechanism for the amplification and persistence of HIF network genes while other acute transcriptional responses to DD subside. Another consideration for the apparent dissonance between substantial epigenome alterations with no transcriptome alterations in $\mathrm{PV}^{-}$cells is the locations of the differential ATAC-seq peaks. In $\mathrm{PV}^{-}$cell types, the vast majority of differential ATAC-seq peaks occur at distal regulatory elements further than 2000 bp away from a TSS ( cortex $=95.9 \%$, striatum $=94.1 \%, \mathrm{GPe}=95.3 \%)$. Changes in accessibility at distal regulatory elements may or may not confer an appreciable change in gene regulation.

Nonetheless, the changes in open chromatin within $\mathrm{GPe} \mathrm{PV}^{+}$ neurons corroborate and strengthen the HIF upregulation that we observed at the transcript level. Namely, HIF family motifs are selectively enriched within DD-increasing ATAC-seq peaks in $\mathrm{GPe} \mathrm{PV}^{+}$neurons. Finally, a higher proportion of GPe $\mathrm{PV}^{+}$ neurons appear to have high Hif2a protein levels in DD tissue compared with healthy tissue. Together, the evidence strongly supports an increase in HIF transcription factor activity in GPe $\mathrm{PV}^{+}$neurons on DD. In the future, cSNAIL could be paired with protein assays to determine the precise HIF protein expression levels, cellular localizations, and DNA binding events in GPe $\mathrm{PV}^{+}$neurons in DD.

In conclusion, cSNAIL is a reliable new strategy for labeling and isolating nuclei of a specific subset of cells within cell typespecific Cre animals. In the case of $\mathrm{GPe} \mathrm{PV}^{+}$neurons in DD mice, cSNAIL enabled targeted transcriptomic and epigenetic interrogation and yielded new cell type-specific results. Most notably, we recovered evidence of HIF oxidative stress signaling that was specific to GPe $\mathrm{PV}^{+}$neurons in this model of DD. These results may help explain the pathogenic progression of DD throughout the basal ganglia and provide hypotheses toward new cell type-specific interventions in PD. We believe a neuron subtype aware approach, such as cSNAIL, will be necessary to tease out molecular correlates of many neurologic and psychiatric disorders, especially where rare neuron populations are heavily implicated.

\section{References}

Bais AS, Kostka D (2019) scds: computational annotation of doublets in single-cell RNA sequencing data. Bioinformatics 36:1150-1158.

Bergman H, Feingold A, Nini A, Raz A, Slovin H, Abeles M, Vaadia E (1998) Physiological aspects of information processing in the basal ganglia of normal and parkinsonian primates. Trends Neurosci 21:32-38.

Bevan MD, Magill PJ, Terman D, Bolam JP, Wilson CJ (2002) Move to the rhythm: oscillations in the subthalamic nucleus-external globus pallidus network. Trends Neurosci 25:525-531.

Blankvoort S, Witter MP, Noonan J, Cotney J, Kentros C (2018) Marked diversity of unique cortical enhancers enables neuron-specific tools by enhancer-driven gene expression. Curr Biol 28:2103-2114.e5. 
Blum D, Torch S, Lambeng N, Nissou M, Benabid AL, Sadoul R, Verna JM (2001) Molecular pathways involved in the neurotoxicity of 6-OHDA, dopamine and MPTP: contribution to the apoptotic theory in Parkinson's disease. Prog Neurobiol 65:135-172.

Botta-or T, Tolosa E, Gelpi E, Sànchez-Pla A, Martí M, Valldeoriola F, Fernández M, Carmona F, Ezquerra M (2012) Neurobiology of disease microarray expression analysis in idiopathic and LRRK2-associated Parkinson's disease. Neurobiol Dis 45:462-468.

Buenrostro JD, Giresi PG, Zaba LC, Chang HY, Greenleaf WJ (2013) Transposition of native chromatin for fast and sensitive epigenomic profiling of open chromatin, DNA-binding proteins and nucleosome position. Nat Methods 10:1213-1218.

Buenrostro JD, Wu B, Chang HY, Greenleaf WJ, Buenrostro JD, Wu B, Chang HY, Greenleaf WJ (2015) ATAC-seq: a method for assaying chromatin accessibility genome-wide. Curr Protoc Mol Biol 109:21.29.121.29.9.

Chamessian A, Youn M, Qadri Y, Berta T, Ji R, Van de Ven T (2018) Transcriptional profiling of somatostatin interneurons in the spinal dorsal horn. Sci Rep 8:6809.

Chan KY, Jang MJ, Yoo BB, Greenbaum A, Ravi N, Wu WL, SánchezGuardado L, Lois C, Mazmanian SK, Deverman BE, Gradinaru V (2017) Engineered AAVs for efficient noninvasive gene delivery to the central and peripheral nervous systems. Nat Neurosci 20:1172-1179.

Chin MH, Qian W, Wang H, Petyuk VA, Bloom JS, Sforza DM, Lac G, Liu D, Khan AH, Cantor RM, Bigelow DJ, Melega WP, Camp DG, Smith RD, Smith DJ (2008) Mitochondrial dysfunction, oxidative stress, and apoptosis revealed by proteomic and transcriptomic analyses of the striata in two mouse models of Parkinson's disease. J Proteome Res 7:666-677.

Day JJ, Sweatt JD (2011) Cognitive neuroepigenetics: a role for epigenetic mechanisms in learning and memory. Neurobiol Learn Mem 96:2-12.

Deal RB, Henikoff S (2010) A simple method for gene expression and chromatin profiling of individual cell types within a tissue. Dev Cell 18:10301040.

Dengler VL, Galbraith M, Espinosa JM (2014) Transcriptional regulation by hypoxia inducible factors. Crit Rev Biochem Mol Biol 49:1-15.

Dutton SB, Makinson CD, Papale LA, Shankar A, Balakrishnan B, Nakazawa K, Escayg A (2013) Preferential inactivation of Scnla in parvalbumin interneurons increases seizure susceptibility. Neurobiol Dis 49:211-220.

Enwright JF, Huo Z, Arion D, Corradi JP, Tseng G, Lewis DA (2018) Transcriptome alterations of prefrontal cortical parvalbumin neurons in schizophrenia. Mol Psychiatry 23:1606-1613.

Ferguson BR, Gao W (2018) PV interneurons: critical regulators of E/I balance for prefrontal cortex-dependent behavior and psychiatric disorders. Front Neural Circuits 12:37.

Fernández-Suárez D, Celorrio M, Lanciego JL, Franco R, Aymerich MS (2012) Loss of parvalbumin-positive neurons from the globus pallidus in animal models of Parkinson disease. J Neuropathol Exp Neurol 71:973982.

Fullard JF, Giambartolomei C, Hauberg ME, Xu K, Voloudakis G, Shao Z, Bare C, Dudley JT, Mattheisen M, Robakis NK, Haroutunian V, Roussos P (2017) Open chromatin profiling of human postmortem brain infers functional roles for non-coding schizophrenia loci. Hum Mol Genet 26:1942-1951.

Griffiths JA, Richard AC, Bach K, Lun AT, Marioni JC (2018) Detection and removal of barcode swapping in single-cell RNA-seq data. Nat Commun 9:2667.

Gu Z, Gu L, Eils R, Schlesner M, Brors B (2014) circlize implements and enhances circular visualization in R. Bioinformatics 30:2811-2812.

Handley A, Schauer T, Ladurner AG, Margulies CE (2015) Designing cell type-specific genome-wide experiments. Mol Cell 58:621-631.

Haque F, Mazzeo D, Patel JT, Smallwood DT, Ellis JA, Shanahan CM, Shackleton S (2010) Mammalian SUN protein interaction networks at the inner nuclear membrane and their role in laminopathy disease processes. J Biol Chem 285:3487-3498.

Hardman CD, Halliday GM (1999) The external globus pallidus in patients with Parkinson's disease and progressive supranuclear palsy. Mov Disord 14:626-633.

Hegeman DJ, Hong ES, Hernandez VM, Chan CS (2016) The external globus pallidus: progress and perspectives. Eur J Neurosci 43:1239-1265.

Heikkila RE, Sieber B, Manzino L, Sonsalla PK (1989) Some features of the nigrostriatal dopaminergic neurotoxin 1-methyl-4-phenyl-1,2,3,6- tetrahydropyridine (MPTP) in the mouse. Mol Chem Neuropathol 10:171-183.

Heiman M, Heilbut A, Francardo V, Kulicke R, Fenster RJ, Kolaczyk ED, Mesirov JP, Surmeier DJ, Cenci MA, Greengard P (2014) Molecular adaptations of striatal spiny projection neurons during levodopa-induced dyskinesia. Proc Natl Acad Sci USA 111:4578-4583.

Herrero M, Estrada C, Maatouk L, Vyas S (2015) Inflammation in Parkinson's disease: role of glucocorticoids. Front Neuroanat 9:1-12.

$\mathrm{Hu}$ H, Gan J, Jonas P (2014) Fast-spiking, parvalbumin ${ }^{+}$GABAergic interneurons: from cellular design to microcircuit function. Science 345: 1255263.

Hunter RL, Dragicevic N, Seifert K, Choi DY, Liu M, Kim H, Cass WA, Sullivan PG, Bing G (2007) Inflammation induces mitochondrial dysfunction and dopaminergic neurodegeneration in the nigrostriatal system. J Neurochem 100:1375-1386.

Inan M, Zhao M, Manuszak M, Karakaya C, Rajadhyaksha AM, Pickel VM, Schwartz TH, Goldstein PA, Manfredi G (2016) Energy deficit in parvalbumin neurons leads to circuit dysfunction, impaired sensory gating and social disability. Neurobiol Dis 93:35-46.

Kann O (2016) The interneuron energy hypothesis: implications for brain disease. Neurobiol Dis 90:75-85.

Khan A, Fornes O, Stigliani A, Gheorghe M, Castro-Mondragon JA, van der Lee R, Bessy A, Chèneby J, Kulkarni SR, Tan G, Baranasic D, Arenillas DJ, Sandelin A, Vandepoele K, Lenhard B, Ballester B, Wasserman WW, Parcy F, Mathelier A (2018) JASPAR 2018: update of the open-access database of transcription factor binding profiles and its web. Nucleic Acids Res 46:D260-D266.

Kim YS, Choi DH, Block ML, Lorenzl S, Yang L, Kim YJ, Sugama S, Cho BP, Hwang O, Browne SE, Kim SY, Hong J, Beal MF, Joh TH (2007) A pivotal role of matrix metalloproteinase-3 activity in dopaminergic neuronal degeneration via microglial activation. FASEB J 21:179-187.

Lake BB, Chen S, Sos BC, Fan J, Kaeser GE, Yung YC, Duong TE, Gao D, Chun J, Kharchenko PV, Zhang K (2018) Integrative single-cell analysis of transcriptional and epigenetic states in the human adult brain. Nat Biotechnol 36:70-80.

Langston JW, Forno LS, Rebert CS, Irwin I (1984) Selective nigral toxicity after systemic administration of 1-methyl-4-phenyl-1,2,5,6-tetrahydropyrine (MPTP) in the squirrel monkey. Brain Res 292:390-394.

Larsen SB, Hanss Z, Krüger R (2018) The genetic architecture of mitochondrial dysfunction in Parkinson's disease. Cell Tissue Res 373:21-37.

Liao Y, Smyth GK, Shi W (2019) The R package Rsubread is easier, faster, cheaper and better for alignment and quantification of RNA sequencing reads. Nucleic Acids Res 47:e47.

Love MI, Huber W, Anders S (2014) Moderated estimation of fold change and dispersion for RNA-seq data with DESeq2. Genome Biol 15:550521.

Lun ATL, Bach K, Marioni JC (2016a) Pooling across cells to normalize single-cell RNA sequencing data with many zero counts. Genome Biol 17:75.

Lun ATL, McCarthy DJ, Marioni JC (2016b) A step-by-step workflow for low-level analysis of single-cell RNA-seq data with Bioconductor. F1000Res 5:2122.

Madisen L, Zwingman TA, Sunkin SM, Oh SW, Hatim A, Gu H, Ng LL, Palmiter RD, Hawrylycz MJ, Allan R, Lein ES, Zeng H (2010) A robust and high-throughput Cre reporting and characterization. Nat Neurosci 13:133-140.

Mallet N, Pogosyan A, Márton LF, Bolam JP, Brown P, Magill PJ (2008) Parkinsonian beta oscillations in the external globus pallidus and their relationship with subthalamic nucleus activity. J Neurosci 28:1424514258.

Mastro KJ, Zitelli KT, Willard AM, Leblanc KH, Kravitz A, Gittis AH (2017) Cell-specific pallidal intervention induces long-lasting motor recovery in dopamine-depleted mice. Nat Neurosci 20:815-823.

Maze I, Shen L, Zhang B, Garcia BA, Shao N, Mitchell A, Sun H, Akbarian S, Allis CD, Nestler EJ (2014) Analytical tools and current challenges in the modern era of neuroepigenomics. Nat Neurosci 17:1476-1490.

McInnes L, Healy J, Melville J (2018) UMAP: uniform manifold approximation and projection for dimension reduction. arXiv:1802.03426.

McLean CY, Bristor D, Hiller M, Clarke SL, Schaar BT, Lowe CB, Wenger AM, Bejerano G (2010) GREAT improves functional interpretation of cis-regulatory regions. Nat Biotechnol 28:495-501. 
McLeay RC, Bailey TL (2010) Motif enrichment analysis: a unified framework and an evaluation on ChIP data. BMC Bioinformatics 11:165.

Miller RM, Kiser GL, Kaysser-Kranich TM, Lockner RJ, Palaniappan C, Federoff HJ (2006) Robust dysregulation of gene expression in substantia nigra and striatum in Parkinson's disease. Neurobiol Dis 21:305-313.

Mo A, Mukamel EA, Davis FP, Luo C, Henry GL, Picard S, Urich MA, Nery JR, Sejnowski TJ, Lister R, Eddy SR, Ecker JR, Nathans J (2015) Epigenomic signatures of neuronal diversity in the mammalian brain. Neuron 86:1369-1384.

Naik S, Larsen SB, Gomez NC, Alaverdyan K, Sendoel A, Yuan S, Polak L, Kulukian A, Chai S, Fuchs E (2017) Inflammatory memory sensitizes skin epithelial stem cells to tissue damage. Nature 550:475-480.

Nott A, Holtman IR, Coufal NG, Schlachetzki JC, Yu M, Hu R, Han CZ, Pena M, Xiao J, Wu Y, Keulen Z, Pasillas MP, O'Connor C, Nickl CK, Schafer ST, Shen Z, Rissman RA, Brewer JB, Gosselin D, Gonda DD, et al. (2019) Brain cell type-specific enhancer-promoter interactome maps and disease-risk association. Science 366:1134-1139.

Ohh M, Park CW, Ivan M, Hoffman MA, Kim TY, Huang LE, Pavletich N, Chau V, Kaelin WG (2000) Ubiquitination of hypoxia-inducible factor requires direct binding to the $\beta$-domain of the von Hippel-Lindau protein. Nat Cell Biol 2:423-427.

Ongwijitwat S, Liang HL, Graboyes EM, Wong-Riley MT (2006) Nuclear respiratory factor 2 senses changing cellular energy demands and its silencing down-regulates cytochrome oxidase and other target gene mRNAs. Gene 374:39-49.

Raudvere U, Kolberg L, Kuzmin I, Arak T, Adler P, Peterson H, Vilo J (2019) g:Profiler: a web server for functional enrichment analysis and conversions of gene lists (2019 update). Nucleic Acids Res 47:W191-W198.

Pietersen CY, Mauney SA, Kim SS, Passeri E, Lim MP, Rooney RJ, Goldstein JM, Petreyshen TL, Seidman LJ, Shenton ME, McCarley RW, Sonntag KC, Woo TU (2014) Molecular profiles of parvalbumin-immunoreactive neurons in the superior temporal cortex in schizophrenia. J Neurogenet 28:70-85.

Pozniak CD, Langseth AJ, Dijkgraaf GJ, Choe Y, Werb Z, Pleasure SJ (2010) Sox10 directs neural stem cells toward the oligodendrocyte lineage by decreasing Suppressor of Fused expression. Proc Natl Acad Sci USA 107:21795-21800.

Rosenberg GA (2009) Matrix metalloproteinases and their multiple roles in neurodegenerative diseases. Lancet Neurol 8:205-216.

Sato M, Tanaka T, Maeno T, Sando Y, Suga T, Maeno Y, Sato H, Nagai R, Kurabayashi M (2002) Inducible expression of endothelial PAS domain protein-1 by hypoxia in human lung adenocarcinoma A549 cells: role of Src family kinases-dependent pathway. Am J Respir Cell Mol Biol 26:127-134.

Saunders A, Huang KW, Sabatini BL (2016) Globus pallidus externus neurons expressing parvalbumin interconnect the subthalamic nucleus and striatal interneurons. PLoS One 11:e0149798.

Saunders A, Johnson CA, Sabatini BL (2012) Novel recombinant adeno-associated viruses for Cre activated and inactivated transgene expression in neurons. Front Neural Circuits 6:47.

Saunders A, Macosko EZ, Wysoker A, Goldman M, Krienen FM, de Rivera H, Bien E, Baum M, Bortolin L, Wang S, Goeva A, Nemesh J, Kamitaki N, Brumbaugh S, Kulp D, McCarroll SA (2018) Molecular diversity and specializations among the cells of the adult mouse brain. Cell 174:10151030.e16.

Schapira AH, Cooper JM, Dexter D, Clark JB, Jenner P, Marsden CD (1990) Mitochondrial complex I deficiency in Parkinson's disease. J Neurochem 54:823-827.

Schindelin J, Arganda-Carreras I, Frise E, Kaynig V, Longair M, Pietzsch T, Preibisch S, Rueden C, Saalfeld S, Schmid B, Tinevez J, White DJ, Hartenstein V, Eliceiri K, Tomancak P, Cardona A (2012) Fiji: an opensource platform for biological-image analysis. Nat Methods 9:676-682.
Schödel J, Oikonomopoulos S, Ragoussis J, Pugh CW, Ratcliffe PJ, Mole DR (2011) High-resolution genome-wide mapping of HIF-binding sites by ChIP-seq. Blood 117:e207-e217.

Schofield CJ, Ratcliffe PJ (2004) Oxygen sensing by HIF hydroxylases. Nat Rev Mol Cell Biol 5:343-354.

Smeyne M, Sladen P, Jiao Y, Dragatsis I, Smeyne RJ (2015) Hifla is necessary for exercise-induced neuroprotection while Hif2a is needed for dopaminergic neuron survival in the substantia nigra pars compacta. Neuroscience 295:23-38.

Soreq L, Ben-Shaul Y, Israel Z, Bergman H, Soreq H (2012) Meta-analysis of genetic and environmental Parkinson's disease models reveals a common role of mitochondrial protection pathways. Neurobiol Dis 45:1018-1030.

Spiegel I, Mardinly A, Gabel H, Bazinet J, Couch C, Tzeng C, Harmin D, Greenber M (2014) Npas4 regulates excitatory-inhibitory balance within neural circuits through cell type-specific gene programs. Cell 157:12161229.

Steullet P, Cabungcal JH, Coyle J, Didriksen M, Gill K, Grace AA, Hensch TK, LaMantia AS, Lindemann L, Maynard TM, Meyer U, Morishita H, O’Donnell P, Puhl M, Cuenod M, Do KQ (2017) Oxidative stress-driven parvalbumin interneuron impairment as a common mechanism in models of schizophrenia. Mol Psychiatry 22:936-943.

Stockwell BR, Friedmann Angeli JP, Bayir H, Bush AI, Conrad M, Dixon SJ, Fulda S, Gascón S, Hatzios SK, Kagan VE, Noel K, Jiang X, Linkermann A, Murphy ME, Overholtzer M, Oyagi A, Pagnussat GC, Park J, Ran Q, Rosenfeld CS, et al. (2017) Ferroptosis: a regulated cell death nexus linking metabolism, redox biology, and disease. Cell 171:273-285.

Sugino K, Hempel CM, Miller MN, Hattox AM, Shapiro P, Wu C, Huang ZJ, Nelson SB (2006) Molecular taxonomy of major neuronal classes in the adult mouse forebrain. Nat Neurosci 9:99-108.

Takada N, Kucenas S, Appel B (2010) Sox10 is necessary for oligodendrocyte survival following axon wrapping. Glia 58:996-1006.

Tasic B (2018) Single cell transcriptomics in neuroscience: cell classification and beyond. Curr Opin Neurobiol 50:242-249.

Traag VA, Waltman L, van Eck NJ (2019) From Louvain to Leiden: guaranteeing well-connected communities. Sci Rep 9:5233.

van Heesbeen HJ, Smidt MP (2019) Entanglement of genetics and epigenetics in Parkinson's disease. Front Neurosci 13:277.

von Bartheld CS, Bahney J, Herculano-Houzel S (2016) The search for true numbers of neurons and glial cells in the human brain: a review of 150 years of cell counting. J Comp Neurol 524:3865-3895.

Wang Z, Gerstein M, Snyder M (2009) RNA-Seq: a revolutionary tool for transcriptomics. Nat Rev Genet 10:57-63.

Wen L, Lu Y, Zhu X, Li X, Woo R, Chen Y, Yin D, Lai C, Terry A, Vazdarjanova A, Xiong WC, Mei L (2010) Neuregulin 1 regulates pyramidal neuron activity via ErbB4 in parvalbumin-positive interneurons. Proc Natl Acad Sci USA 107:1211-1216.

West AE, Chen WG, Dalva MB, Dolmetsch RE, Kornhauser JM, Shaywitz AJ, Takasu MA, Tao X, Greenberg ME (2001) Calcium regulation of neuronal gene expression. Proc Natl Acad Sci USA 98:11024-11031.

Whitney O, Pfenning AR, Howard JT, Blatti CA, Liu F, Ward JM, Wang R, Audet JN, Kellis M, Mukherjee S, Sinha S, Hartemink AJ, West AE, Jarvis ED (2014) Core and region-enriched networks of behaviorally regulated genes and the singing genome. Science 346:1256780.

Willard AM, Isett BR, Whalen TC, Mastro KJ, Ki CS, Mao X, Gittis AH (2019) State transitions in the substantia nigra reticulata predict the onset of motor deficits in models of progressive dopamine depletion in mice. Elife 8:e42746.

Wolf FA, Angerer P, Theis FJ (2018) SCANPY: large-scale single-cell gene expression data analysis. Genome Biol 19:15.

Xie W, Chung KK (2012) Alpha-synuclein impairs normal dynamics of mitochondria in cell and animal models of Parkinson's disease. J Neurochem 122:404-414. 